\title{
Precision of multi-frequency electronic apex locators
}

\section{Abstracted from \\ Tsesis I, Blazer T, Ben-Izhack G, et al.}

The Precision of Electronic Apex Locators in Working Length Determination:

A Systematic Review and Meta-analysis of the Literature. J Endod 2015; 41: 1818-1823.

Address for correspondence: Dr Igor Tsesis, Department of Endodontology, Maurice and Gabriela Goldschleger, School of Dental Medicine, Tel Aviv University, Tel Aviv, Israel. E-mail: dr.tsesis@gmail.com

\section{Question: How precise are electronic apex locators in identifying the apical constriction?}

Data sources Cochrane Central Register of Controlled Trials, Medline, Embase and Scopus databases.

Study selection Studies that reported the precision of electronic apex locators (EALs) in locating the apical constriction (AC) in primary root canal treatment of human teeth compared with a histologic evaluation of the AC were considered.

Data extraction and synthesis Data were extracted and quality assessed independently by two reviewers.

Results Ten studies were included, reporting on $1105 \mathrm{EAL}$ measurements. Seven studies were considered to be at high risk of bias and three at low risk. Four different EALs were evaluated; Root ZX (J Morita, Tokyo, Japan), Justy II (Hager \& Werken GmbH \& Co, Duisburg, Germany), Endy 5000 (Loser Co, Leverkusen, Germany) and Endox (Lysis Co, Milan, Italy). Three EALs, Root ZX, Justy II and Endy 5000 were more accurate than the Endox in determining the distance between the file tip and the apical constriction. Pulp status was only available for 194 (17.55\%) of the measurements. The status of the pulp (vital or necrotic) had no significant effect on precision. Conclusions The precision of electronic working length measurement depends on the device used and the type of irrigation and is not influenced by the status of the pulp tissue.

\section{Commentary}

The precise location of the working distance during root canal treatment is important to not only ensure that the root canal system is free of necrotic tissue and microorganisms, but also to ensure that biological and mechanical objectives of root canal preparation are met. ${ }^{1}$

It is well acknowledged that defining the canal terminus is problematic $^{2}$ and hence the minor apical foramen is considered clinically as a consistent anatomical feature. ${ }^{3}$ The lack of a proper definition for the termination of the root canal preparation has led to significant variability in reporting electronic apex locators (EAL) accuracies. ${ }^{4-7}$ This study defined the ability of EAL to locate the histologic minor foramen as the gold standard; an important step in standardisation.

This manuscript looked at the precision of multi-frequency EAL in establishing length using a meta-analysis. The quality of the ten studies included in this meta-analysis were categorised as, 'low risk of bias' and 'high risk of bias' based on specific quality criteria. The authors stated that bias is a systematic error, or deviation from the truth, that could lead to a false estimation of the intervention effect. Despite seven of the included studies being classified as 'high risk of bias,' the authors failed to clarify the inclusion of the associated data into the meta-analysis.

It is clear from literature that the use of multiple frequencies by EAL manufacturers allows for calibration of variables (presence of fluid, tissue, type of fluid etc) that could affect the determination of working lengths. ${ }^{8-10}$ This study highlights the variations that could result from the electroconductivity of irrigant solutions. 9,10 To some extent, modern apex locators with multiple frequencies account for these electroconductive variations, however, it is important to ensure that manufacturers' recommendations are followed, for example, the manufacturer of CanalPro ${ }^{\mathrm{TM}}$ (Coltene® Endo, $\mathrm{OH}, \mathrm{USA})$ units indicate that $\mathrm{NaOCl}$ irrigation above $3 \%$ is not recommended. ${ }^{11}$

Pommer et al. (2002) reported that a five signal frequencies EAL with the ability to work effectively in the presence of electrolytes was less effective in necrotic canals when compared to vital canals. ${ }^{12}$ This systematic review found no significant difference between vital and necrotic teeth in the precision of EALs.

Despite the authors applying stringent inclusion and exclusion criteria to avoid misrepresentation of data, it should be noted that a lack of understanding of the variability in description of EAL output reading between manufacturers might result in differences in outcome. Morita ${ }^{\mathrm{TM}}$, for example, indicates that the Root ZX 
II $^{\mathrm{TM}}$ (Morita, Japan) meter reading at 0.5 indicates that the tip of the file is at the apical constriction, ${ }^{13}$ while others may indicate that the apical constriction is reached at ' 0 '.${ }^{11}$ Furthermore, some manufacturers indicate that no numerical significance (in $\mathrm{mm}$ ) be placed on the meter gauge reading ${ }^{13}$ while others like Apex ID ${ }^{\mathrm{TM}}$ (Sybron Endo, CA, USA) do calibrate for changes in length in mm. ${ }^{14}$ It should be noted that manufacturers may use terms such as 'apical foramen', 'root apex', 'apical constriction', 'physiological foramen' etc interchangeably or as stand-alone; making appropriate and accurate interpretation of EAL working length (WL) determination confusing. ${ }^{14}$ Haffner et al. (2005) when reporting the accuracy of electronic apex locators in an in vivo study failed to elucidate the difference in display reading of the EAL used as reference. ${ }^{15}$

Notwithstanding the ability of modern apex locators to determine the WL with significant accuracy, it seems prudent to substantiate EAL outputs visually using radiographs whenever possible prior to preparation of root canals to the working length.

Roy George School of Dentistry and Oral Health, Griffith University, Gold Coast, Australia
1. Schilder H. Cleaning and shaping the root canal. Dent Clin North Am 1974; 18: 269-296

2. Dummer PM, McGinn JH, Rees DG. The position and topography of the apical canal constriction and apical foramen. Int Endod / 1984; 17: 192-198.

3. Katz A, Tamse A, Kaufman AY. Tooth length determination: a review. Oral Surg Oral Med Oral Path 1991; 72: 238-242.

4. Lucena-Martin C, Robles-Gijon V, Ferrer-Luque CM, de Mondelo JM. In vitro evaluation of the accuracy of three electronic apex locators. J Endod 2004; 30: 231-233.

5. Jenkins JA, Walker WA 3rd, Schindler WG, Flores CM. An in vitro evaluation of the accuracy of the root ZX in the presence of various irrigants. J Endod 2001; 27: 209-211.

6. Azabal M, Garcia-Otero D, de la Macorra JC. Accuracy of the Justy II Apex locator in determining working length in simulated horizontal and vertical fractures. Int Endod / 2004; 37: 174-177.

7. Hoer $D$, Attin $T$. The accuracy of electronic working length determination. Int Endod J 2004; 37: 125-131.

8. Gordon MP, Chandler NP. Electronic apex locators. Int Endod J 2004; 37: 425-437.

9. Fan W, Fan B, Gutmann JL, Bian Z, Fan MW. Evaluation of the accuracy of three electronic apex locators using glass tubules. Int Endod / 2006; 39: 127-135.

10. Mull JP, Manjunath V, Manjunath M. Comparison of accuracy of two electronic apex locators in the presence of various irrigants: An in vitro study. J Conserv Dent 2012; 15: 178-182.

11. Coltene Endo. CanalProTM apex locator user's guide. 2014. Available from: www. coltene.com/fileadmin/Data/EN/Products/Endodontics/Root_Canal_Shaping/ CANALPRO_APEX_LOCATOR/Apex_Locator_NAM_IFU.pdf [Accessed 4 September 2016]

12. Pommer O, Stamm O, Attin T. Influence of the canal contents on the electrical assisted determination of the length of root canals. J Endod 2002; 28: 83-85.

13. Morita. Root ZX II operation manual. 2007. Available from: http://global.morita.com/ usa/root/img/pool/pdf/ifu_msds/rootzx_ii_apex_ifu_dp-rcm_m8037-ea-2-v3.pdf [Accessed 4 September 2016]

14. Sybron Endo. Apex ID instruction manual. 2016. Available from: http://www.profident. $\mathrm{pl} /$ media/142960/apex_id_instrukcja_01.21.13_.pdf [Accessed 4 September 2016]

15. Haffner C, Folwaczny M, Galler K, Hickel R. Accuracy of electronic apex locators in comparison to actual length - an in vivo study. J Dent 2005; 33: 619-625. 